\title{
Dederer, Hans-Georg/Shin, Yu-Cheol (Hrsg.), Künstliche Intelligenz und juristische Herausforderungen.
}

\author{
Mohr Siebeck Verlag, Tübingen, 2021. XVI, 170 Seiten. ISBN \\ 978-3-16-160048-7
}

\section{Christian Armbrüster}

Angenommen: 30. April 2021 / Online publiziert: 31. Mai 2021

(C) Der/die Autor(en) 2021

Die Künstliche Intelligenz (KI) ist auch im Versicherungssektor mehr und mehr in den Blick gelangt, und mit ihr sind dies die damit verbundenen Rechtsfragen. Die vorzustellende Neuerscheinung, welche die Beiträge einer Tagung versammelt, deckt ein breites Themenspektrum ab. Dieses reicht von Bürgerlichen Recht über das Arbeits- und das Urheberrecht bis hin zum Strafprozessrecht und zum Völkerrecht. Für die versicherungsrechtlich interessierte Leserschaft verdient der Beitrag von Thomas Rüfner (S. 15-42) besondere Aufmerksamkeit. Darin geht es um juristische Herausforderungen der KI aus der Perspektive des Privatrechts. Darauf soll im Folgenden näher eingegangen werden.

Rüfner setzt sich zunächst mit der Definition von KI auseinander, die durch die „High Level Group on Artificial Intelligence“ der Europäischen Kommission formuliert worden ist. Diese Definition (abrufbar unter https://digital-strategy.ec.europa. eu/en/library/definition-artificial-intelligence-main-capabilities-and-scientific-disci plines) lautet wie folgt:

Artificial intelligence (AI) systems are software (and possibly also hardware) systems designed by humans that, given a complex goal, act in the physical or digital dimension by perceiving their environment through data acquisition, interpreting the collected structured or unstructured data, reasoning on the knowledge, or processing the information, derived from this data and deciding the best action(s) to take to achieve the given goal. AI systems can either use symbolic rules or learn a numeric model, and they can also adapt their behaviour by analysing how the environment is affected by their previous actions.

Christian Armbrüster $(\bowtie)$

Fachbereich Rechtswissenschaft, Freie Universität Berlin, Van't-Hoff-Str. 8, 14195 Berlin,

Deutschland

E-Mail: c.armbruester@fu-berlin.de 
As a scientific discipline, AI includes several approaches and techniques, such as machine learning (of which deep learning and reinforcement learning are specific examples), machine reasoning (which includes planning, scheduling, knowledge representation and reasoning, search, and optimization), and robotics (which includes control, perception, sensors and actuators, as well as the integration of all other techniques into cyber-physical systems).

Diese Definition ist im Schrifttum in verschiedener Hinsicht auf Kritik gestoßen. Soweit gegen sie eingewandt wird, dass sie die Möglichkeit von Systemen unbeachtet lasse, die sich ihre Ziele selbst setzen, hält Rüfner (S. 17f.) dies nicht für stichhaltig, da diese sog. starke KI derzeit nicht existiere. Dies trifft zu; freilich wird man insoweit die weitere technologische Entwicklung im Blick zu behalten haben. Gewichtiger erscheint dem Autor der zweite Kritikpunkt, nämlich dass die existierenden Systeme bislang nicht immer die optimale Lösung (in der Definition: „,best action[s]“") der gestellten Aufgabe finden. Dieser Kritik stimmt Rüfner mit der Begründung zu, dass die KI-Systeme keineswegs fehlerlos arbeiteten, sondern dass sie (lediglich) darauf angelegt seien, optimale Entscheidungen zu treffen. Man wird freilich die Definition ohnehin in diesem einschränkenden Sinne zu verstehen haben. Davon unabhängig ist die Diskussion jedenfalls deshalb interessant, weil sie den Blick auf die mögliche Fehlerhaftigkeit der durch ein KI-System getroffenen Entscheidungen lenkt; gerade hier beginnen die rechtlichen Probleme.

Im Bemühen um eine juristisch tragfähige Definition macht Rüfner als Unterscheidungsmerkmal gegenüber schlichten Computerprogrammen die Lernfähigkeit von KI-Systemen aus (S. 18). Problematisch sei allerdings, dass die verschiedenen Erscheinungsformen des Maschinellen Lernens es dem Menschen nur schwer ermöglichen vorauszusagen oder nachzuvollziehen, ob und auf welche Weise die trainierte Maschine zu richtigen Ergebnissen gelangt. Aus versicherungsrechtlicher Sicht ist dies insbesondere im Hinblick auf die von den Aufsichtsbehörden geforderte Nachvollziehbarkeit der dem Versicherer zuzurechnenden Entscheidungen (etwa zur Antragsannahme oder zur Regulierung) bedeutsam.

Im Streit um die Frage, ob durch KI gesteuerte Maschinen als rechtsfähig anzusehen sein sollten (,E-Person“), äußert der Autor sich ablehnend zu der dies befürwortenden Entschließung des Europäischen Parlaments von 2017 (S. 20f., s. auch S. 27; zur Diskussion s. ferner Teubner, AcP 218 [2018], 155 ff.). In der Tat handelt es sich bei diesen Systemen allein um ein neuartiges Hilfsmittel, und die Gefahr von Kontrollverlusten bestand auch bei früheren technologischen Neuerungen. Hinzufügen lässt sich, dass mit Blick auf Haftungsfragen die Schaffung einer E-Person von vornherein nur in Betracht käme, wenn ihr ein hinreichender Haftungsfonds zugeordnet würde. Dann liegt es aber näher, sogleich diejenigen natürlichen oder juristischen Personen in Anspruch zu nehmen, welche sich der entsprechenden Systeme bedienen.

Sodann befasst sich Rüfner mit einigen praxisrelevanten Streitfragen zur Anwendung des BGB auf KI-Systeme. Ein besonders umstrittenes Problemfeld bieten hier die eng miteinander verbundenen Fragen der Zurechnung von mittels KI abgegebenen Willenserklärungen und des Anfechtungsrechts. Als Grundsatz kann festgehalten werden, dass sich der Verwender das Handeln einer Software zurechnen lassen muss, 
da er das System in seiner Risikosphäre einsetzt. Allerdings überlässt er bei durch KI gesteuerten Systemen die Entscheidungsverantwortung umfassend dem System; er trägt damit das Risiko für das Ergebnis, ohne dass er in den Systemprozess eingreifen könnte. Rüfner geht zutreffend davon aus, dass die Zurechnung dadurch nicht in Frage gestellt wird (S. 22 f.).

Was die Anfechtbarkeit von durch das System erstellten und geäußerten Willenserklärungen angeht, wendet Rüfner sich dagegen, die Regeln über Blanketterklärungen sinngemäß anzuwenden, so dass von einem unbeachtlichen Motivirrtum auszugehen wäre. Demgegenüber will er eine Parallele zu den Fällen ziehen, in denen jemand eine Vertragsurkunde ungelesen unterschreibt (S. 25f.). Folgt man dem, so ist die Anfechtung nur in den (in der Praxis seltenen) Fällen ausgeschlossen, in denen der Verwender des KI-Systems sich über den Inhalt der Erklärung überhaupt keine Vorstellung macht. Diese weitgehende Eröffnung eines Anfechtungsrechts nach § 119 Abs. 1 BGB (verbunden mit der Pflicht zum Ersatz des Vertrauensschadens nach $\S 122$ BGB) erscheint für den von Rüfner gebildeten Beispielsfall nachvollziehbar, in dem das System das Foto eines Kaufgegenstands fehlerhaft mit einer Bestellung verknüpft. Anders ist die Lage indessen dann, wenn das System fehlerfrei funktioniert, d.h. die objektiv richtige Entscheidung trifft, auf die jedoch der Wille des Verwenders nicht gerichtet war. In solchen Fällen erscheint es vorzugswürdig von einem im Vorfeld des KI-Einsatzes liegenden unbeachtlichen Motivirrtum darüber auszugehen, dass das System eine Entscheidung nach dem Willen des Verwenders treffen werde (MünchKomm-BGB/Armbrüster, 9. Aufl. 2021, § 119 Rn. 51 [im Druck]).

Auch das weitere Themenfeld der Haftung für KI-gesteuerte Roboter spricht Rüfner an (S. $29 \mathrm{ff}$.). Hierzu legt er dar, dass die Haftungsfrage sich in der Praxis in vielen Fällen nicht als problematisch erweise. Dies sei außer im Bereich vertraglicher Haftung für die Erfüllung der primären Leistungspflicht (einschließlich Gewährleistungsansprüchen) auch dann der Fall, wenn die Herstellerhaftung nach dem ProdHaftG oder nach den Regeln der Produzentenhaftung gem. $§ 823$ Abs. 1 BGB eingreift (S. 30f.). Eine Haftungslücke bestehe mithin allein in solchen Fällen, in denen es weder um das Erfüllungsinteresse geht noch ein Produktfehler des KISystems den Schaden verursacht hat. Insoweit sei es weiterhin anzuerkennen, dass eine allgemeine verschuldensunabhängige Haftung des Halters von Sachen nicht besteht. Zu einem unerträglichen Zustand führe dies nicht (S. 32). Dies lässt sich aus heutiger Sicht hören, wobei freilich die rechtspolitischen Diskussion um eine Ausdehnung der Halterhaftung auf Bereiche jenseits des Kfz-Verkehrs (zu ihm s. sogleich) damit noch keineswegs abgeschlossen sein dürfte.

Was die verschuldensunabhängige Haftung des Kfz-Halters im Straßenverkehr nach $\S 7$ StVG angeht, legt Rüfner zutreffend dar, dass es sich bei einer Fehlfunktion automatisierter Einrichtungen wie etwa eines Bremsassistenten nicht um höhere Gewalt i.S.v. $\$ 7$ Abs. 2 StVG handelt, wodurch die Gefährdungshaftung ausgeschlossen würde (S. 34). Auch das autonome Fahren erfordere keine Abkehr von der Gefährdungshaftung des Halters (S. 35). Diese Einschätzung verdient Zustimmung. Aus versicherungsrechtlicher Warte ist zu ergänzen, dass sich das System von Halterhaftung und Pflicht-Haftpflichtversicherung im Interesse eines effektiven 
Schutzes von Verkehrsopfern bewährt hat (näher Armbrüster, in: FS Ch. Huber, 2020, S. 27 ff.).

Einen weiteren für das Versicherungsrecht relevanten Themenkreis spricht Rüfner an, indem er abschließend auf die Gefahr diskriminierenden Verhaltens durch KISysteme zu sprechen kommt. Dabei greift er zur Illustration den - in einem PraxisExperiment tatsächlich vorgekommenen - Fall eines Chatbots auf, der anhand von Daten aus sozialen Netzwerken gelernt hat, rassistische Äußerungen vorzunehmen. Der Autor bejaht Beseitigungs- und Unterlassungsansprüche analog $§ 1004$ BGB, während er einen verschuldensabhängigen Entschädigungsanspruch aus $\S 823$ Abs. 1 oder $\S 831$ BGB letztlich verneint (S. 36 ff.). Die Thematik zeigt, dass es für einen sinnvollen Einsatz der Lernfunktion von KI entscheidend darauf ankommt, mit welchem Datenmaterial gearbeitet wird.

Die weiteren Beiträge erscheinen aus versicherungswissenschaftlicher Perspektive vergleichsweise weniger relevant; gleichwohl bieten auch sie eine interessante Lektüre. Dies gilt insbesondere für die Ausführung zu Smart Contracts (S. $43 \mathrm{ff}$.; Jin) und zum Einsatz von KI in der Arbeitswelt (S. 63 ff.; Chin). Der gut lesbare Band kann daher auch insoweit zur Lektüre empfohlen werden.

Funding Open Access funding enabled and organized by Projekt DEAL.

Open Access Dieser Artikel wird unter der Creative Commons Namensnennung 4.0 International Lizenz veröffentlicht, welche die Nutzung, Vervielfältigung, Bearbeitung, Verbreitung und Wiedergabe in jeglichem Medium und Format erlaubt, sofern Sie den/die ursprünglichen Autor(en) und die Quelle ordnungsgemäß nennen, einen Link zur Creative Commons Lizenz beifügen und angeben, ob Änderungen vorgenommen wurden.

Die in diesem Artikel enthaltenen Bilder und sonstiges Drittmaterial unterliegen ebenfalls der genannten Creative Commons Lizenz, sofern sich aus der Abbildungslegende nichts anderes ergibt. Sofern das betreffende Material nicht unter der genannten Creative Commons Lizenz steht und die betreffende Handlung nicht nach gesetzlichen Vorschriften erlaubt ist, ist für die oben aufgeführten Weiterverwendungen des Materials die Einwilligung des jeweiligen Rechteinhabers einzuholen.

Weitere Details zur Lizenz entnehmen Sie bitte der Lizenzinformation auf http://creativecommons.org/ licenses/by/4.0/deed.de. 